\title{
Desarrollo de estructuras osteointegrables base titanio para implantes biomédicos mediante tecnologías de impresión 3D y pulvimetalurgia.
}

Development of osteointegrable structures based on titanium for biomedical implants using 3D printing technologies and powder metallurgy.

Presentación: 22/11/2019

\section{Doctorando:}

\section{César Andrés Roure}

Pertenencia: Universidad Tecnológica Nacional, Facultad Regional Córdoba, Laboratorio del Dpto. de Ingeniería Metalúrgico cesararoure@chotmail.com.ar

\section{Director/es:}

\section{Carlos Rodolfo Oldani \\ Roberto Oscar Lucci}

\begin{abstract}
Resumen
En el siguiente trabajo se plantea la utilización de la tecnología de impresión 3D para prototipado de moldes con la geometría definida de la prótesis, combinando técnicas de moldeo por gel-casting en la preparación de un producto en verde fabricado con polvo de $\mathrm{TiH}_{2}$ que permita llenar la cavidad del molde impreso en 3D y posterior sinterización controlada, se logrará la obtención de implantes biomédicos de titanio con la rugosidad y porosidad adecuada para la oseointegración.
\end{abstract}

Palabras claves: Gel-casting, impresión 3D, biomateriales, prototipado.

\begin{abstract}
The following work proposes the use of 3D printing technology for prototyping of molds with the defined geometry of the prosthesis, combining gel-casting molding techniques in the preparation of a green product made of $\mathrm{TiH} 2$ powder that allows filling The cavity of the 3D printed mold and subsequent controlled sintering will achieve the achievement of biomedical titanium implants with the roughness and porosity suitable for osseointegration.
\end{abstract}

Keywords: Gel-casting, 3D printing, biomaterials, prototyping.

\section{Introducción}

El aumento en la edad de los seres humanos, trae aparejada la degradación del hueso, su enfermedad o trauma. Estos problemas necesitan ser resueltos mediante el uso de dispositivos permanentes, temporales o biodegradables. En la actualidad, los médicos especialistas prefieran mantener el método clásico de implantes cementados. Sin embargo, la fijación biológica usando el crecimiento de nuevo hueso, formado en las no-uniformidades de la superficie del implante, es más adecuado desde el punto de vista de un reemplazo óseo a largo plazo [1]. La elección del titanio como material de implante con fijación biológica se debe a sus principales características biomédicas, como el tener muy buenas propiedades mecánicas, ser cuasi-bioinerte y poseer buena resistencia a la corrosión comparado con otros metales y aleaciones [2]. Por otro lado, la incorporación de la tecnología de impresión 3D abre una gran puerta a nuevos métodos de fabricación de prótesis, ya que permite obtener prótesis altamente personalizadas a través del diseño por CAD-CAM y con geometrías complejas y terminación superficial controlada. Sin embargo, la tecnología de impresión 3D en metales para aplicaciones directas requiere métodos como el 
sinterizado selectivo por láser o proyección de electrones, procesos extremadamente costosos y de larga duración [3, 4].

En este trabajo se investigó la utilización de la tecnología de impresión 3D con materiales poliméricos para generar un prototipado del molde o negativo de una prótesis. Posteriormente, el molde es llenado con el material que confeccionará finalmente la prótesis. Como material de relleno de los moldes, se planteó el uso de polvos de hidruro de titanio $\left(\mathrm{TiH}_{2}\right)$ combinados con la técnica de preparación de la pieza en verde por gel-casting. Una vez obtenida la pieza o prótesis en verde, se efectuó la sinterización en vacío para conseguir el producto metálico base titanio con la geometría final de la prótesis.

\section{Resultados}

Las tareas realizadas se reflejan en el siguiente diagrama de flujo de Figura 1:

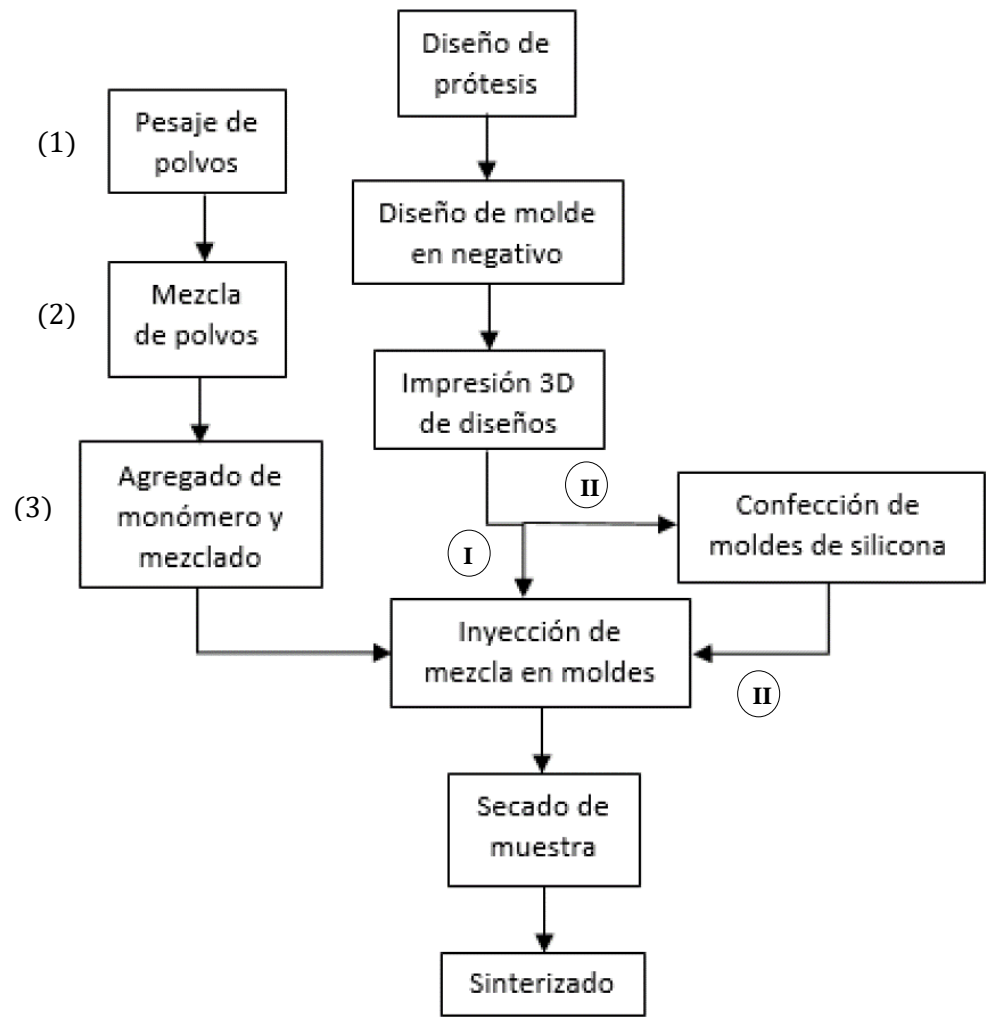

Figura 1 - Flow sheet de proceso

Se efectuó una primera etapa de confección de un modelo de prótesis parcial de cadera (tallo femoral), diseñado en un software CAD en una escala reducida 1:2 del tamaño de prótesis para personas adultas (Figura 2). Basándonos en este diseño, se estudió y generó los diseños de moldes en negativos a través de CAD, adecuando al proceso de moldeo por gel-casting. Para esto se plantearon dos modelos; por un lado, un modelo de inyección directa de material, y por el otro, uno de inyección indirecta, donde se fabricó el negativo de la pieza, para fabricar un molde de caucho siliconado. Una vez finalizada la etapa de diseño, se estudiaron las condiciones óptimas para la ejecución de la impresión 3D (material, altura de capa, velocidad de impresión, densidad de llenado del modelo, temperatura de cama, temperatura de extrusor, diámetro de boquilla) para cada uno de los modelos propuestos (Tabla 1 - Parámetros de impresión para modelos "I" y "II"Tabla 1).

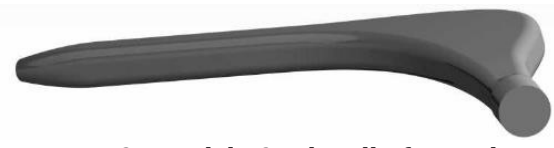

Figura 2 - Modelo 3D de tallo femoral. 


\begin{tabular}{|c|c|c|c|c|c|c|c|}
\hline Modelo & Material & $\begin{array}{c}\text { Altura } \\
\text { de capa } \\
{[\mathrm{mm}]}\end{array}$ & $\begin{array}{c}\text { Vel. de } \\
\text { impresión } \\
{[\mathrm{mm} / \mathrm{s}]}\end{array}$ & $\begin{array}{c}\text { Densidad } \\
\text { de llenado } \\
{[\%]}\end{array}$ & $\begin{array}{c}\mathrm{T}^{\circ} \\
\text { de } \\
\text { cama } \\
{\left[{ }^{\circ} \mathrm{C}\right]}\end{array}$ & $\begin{array}{c}\mathrm{T}^{\circ} \text { de } \\
\text { extrusor } \\
{\left[{ }^{\circ} \mathrm{C}\right]}\end{array}$ & $\begin{array}{c}\text { Diámetro } \\
\text { de boquilla } \\
{[\mathrm{mm}]}\end{array}$ \\
\hline I & PLA & 0,1 & 60 & 15 & 62 & 195 & 0,4 \\
\hline II & PLA & 0,1 & 60 & 25 & 62 & 195 & 0,4 \\
\hline
\end{tabular}

El material de relleno para estos modelos se obtuvo a partir de: (1) pesaje de los polvos en proporcione de hidruro de titanio y resina acrílica iguales a $91 \% \mathrm{TiH}_{2}$ - $9 \% \mathrm{RA}$; (2) pre-mezclado para obtener un producto homogéneo en distribución de partículas; (3) agregado del monómero a la pre-mezcla y posterior mezclado para obtener un material de relleno pastoso capas de ser inyectado ("slurry"). Una vez obtenidos de moldes, se procedió a inyectar la mezcla de relleno mediante jeringas de uso medicinal y se aseguró el correcto llenado de los mismos. Los resultado obtenidos para cada modelo, luego de 24 hs de secado a $\mathrm{T}^{\circ}$ ambiente, se muestran en las Figura 3 y Figura 4 .

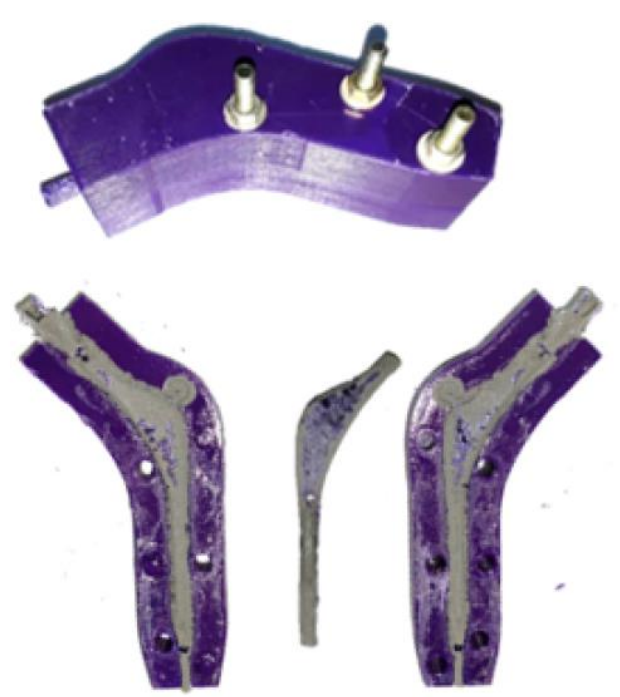

Figura 3 - Muestra 1, fabricada a través de molde de inyección directa (Modelo I).

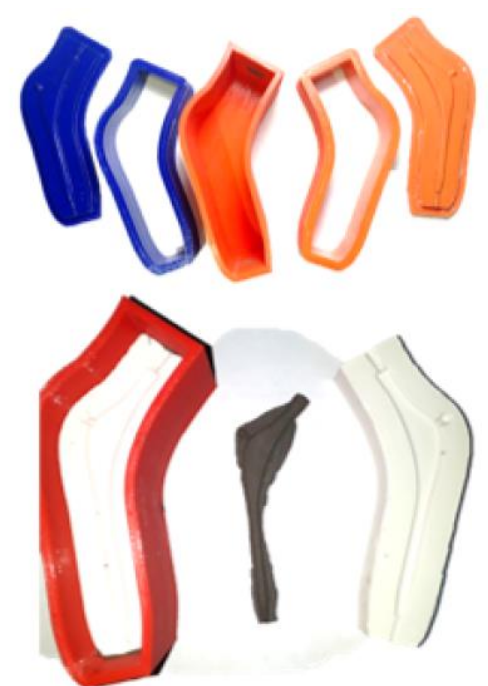

Figura 4 - Muestra 2, fabricada a través de molde de caucho siliconado (Modelo II).

Finalmente, se sometió a las piezas obtenidas a un proceso de sinterizado a elevada temperatura y en atmosfera controlado (presión $=10^{-6} \mathrm{mmHg}$ ). El ciclo de sinterizado utilizado se observa en la Figura 5 . 


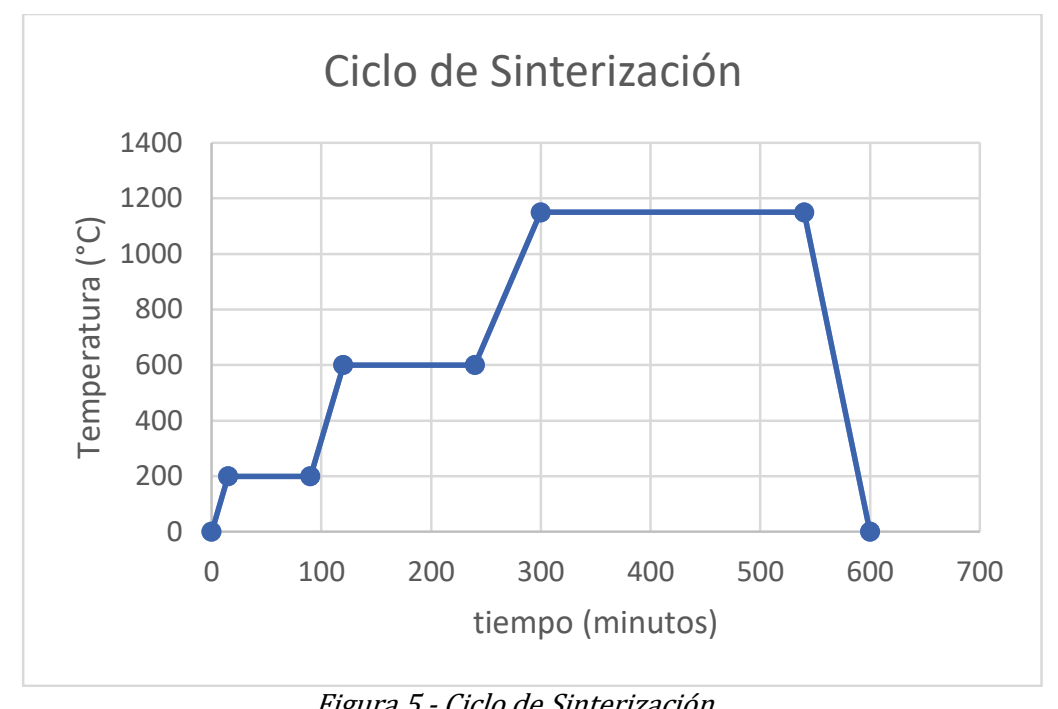

Figura 5 - Ciclo de Sinterización

\section{Análisis Macrográfico}

La muestra 1 presentó dilatación en su sección transversal y una fisura que recorre la sección longitudinal (1). Además, se produjeron dos fracturas durante el sinterizado (2). Estos defectos podrían ser asociados a un moldeo incorrecto. Por otra parte, la muestra 2 reflejó una contracción generalizada, principalmente en la sección de mayor cantidad de material (3) y puede ser asociado al proceso de sinterización o al moldeo previo y deben estudiarse las condiciones que disminuyan dicha contracción (ver Figura 6 y Figura 7).

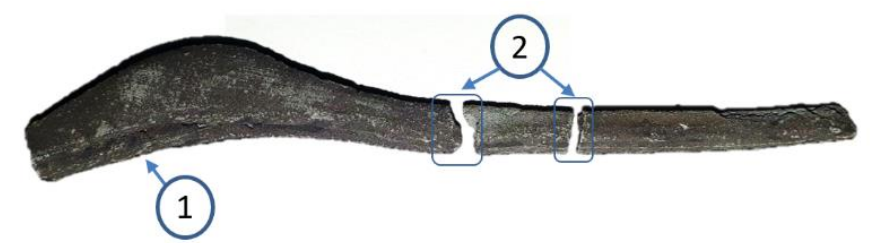

Figura 6 - Muestra 1 (post sinterizado).

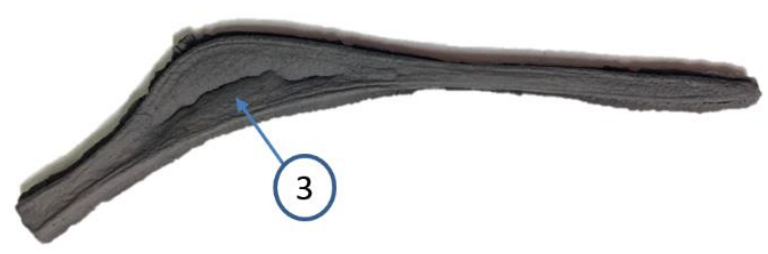

Figura 7 - Muestra 2 (post sinterizado).

\section{$\underline{\text { Análisis Micrográfico }}$}

La matriz de Ti reveló una sinterización eficiente para ambas muestras y se observa una distribución homogénea de la porosidad, sin embargo, la cantidad de poros presentes varía para cada parte del tallo femoral (Figura 8), como se muestra en la Tabla 2 - Micrografías de muestras 1 y 2 en zonas A, B y C.Tabla 2 a continuación: 


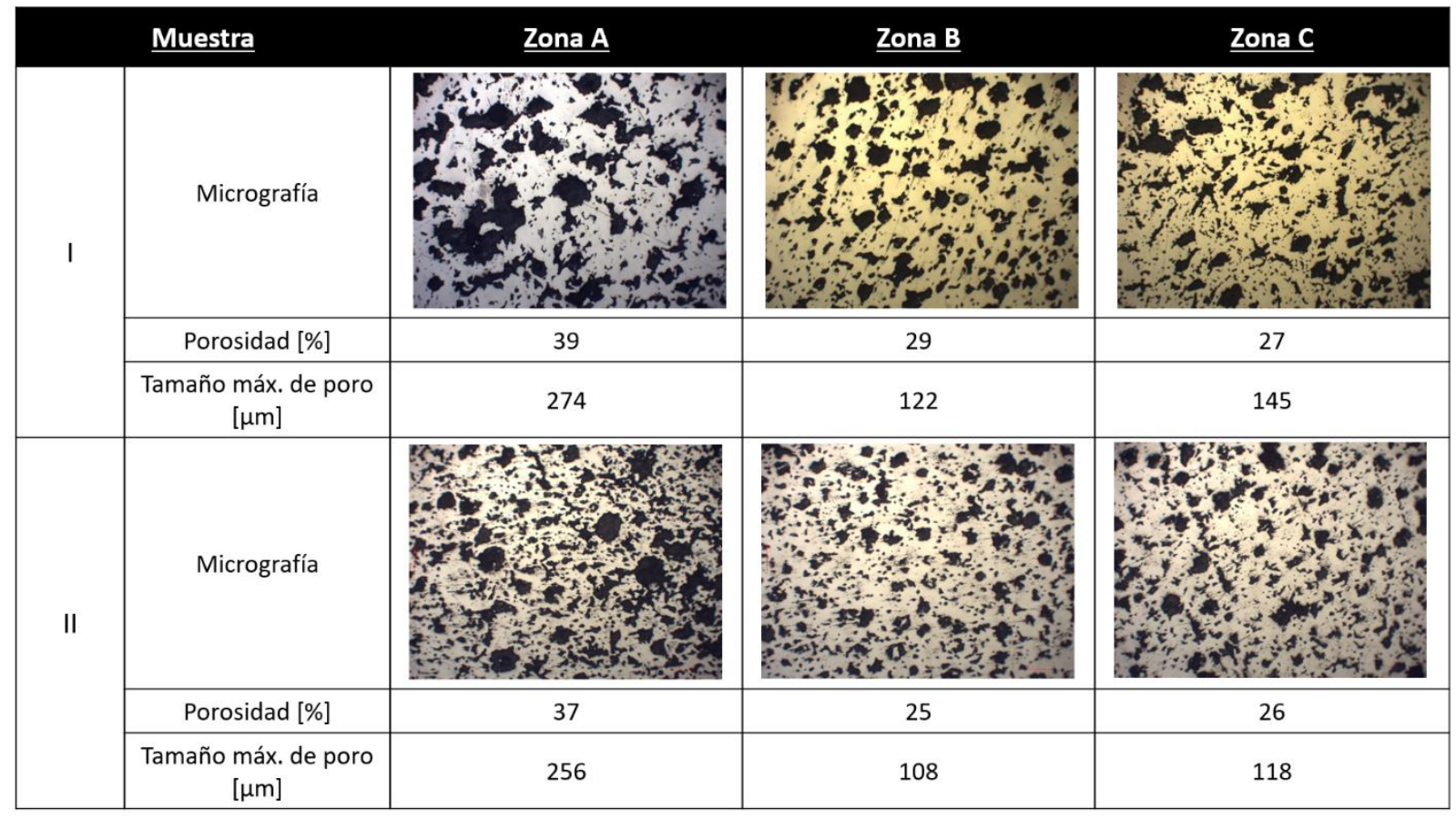

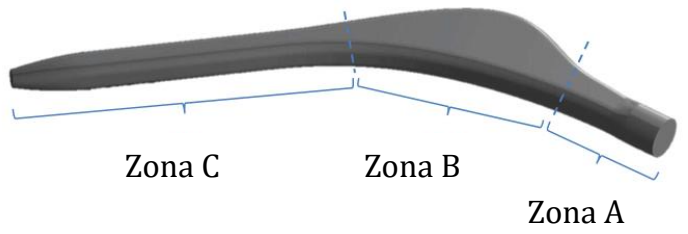

Figura 8 - Identificación de las zonas A, B y C para análisis micrográfico.

\section{Conclusiones}

Se determinó que es posible el desarrollo de prototipos mediante CAD-CAM e impresión 3D de polímeros para fabricación de moldes de prótesis biomédicas. Por otra parte, se consiguió obtener una pieza metálica, pero con distorsión geométrica y en la terminación superficial que evidenció presencia de poros distribuidos heterogéneamente, posiblemente por los gases generados durante la inyección de las piezas junto a la volatilización del monómero en la etapa de sinterización. En general, el tamaño máximo de poros estuvo comprendido entre valores $108 \mu \mathrm{m}$ y $274 \mu \mathrm{m}$, valores los cuales se encuentran próximos de los valores recomendados para favorecer una correcta oseointegración.

\section{Referencias}

[1] K. Mallick. (2014). Bone Substitute Biomaterials. Ed. Elsevier, pp. 156-157.

[2] R. M. López Padilla. Desarrollo de estructuras porosas de titanio biocompatible, obtenidas por pulvimetalurgia, Tesis Doctoral, FCEFyN-UNC, Córdoba, Argentina, 2013.

[3] A. Miyer Suárez Castrillón et. al. Aplicación de herramientas CAD/CAM para el diseño y fabricación de prototipos de moldes de inyección de plásticos, Tecnura, 19 No. 46 (2015) 115-121.

[4] J. Horvath, R. Cameron. 3D Printed Science Projets Volume 2. Physics, Math, Engineering, and Geology Models, 2017, California, USA. 